\title{
Does She Advance Her Development in The Face of Cancer? A Structural Equation Model of Posttraumatic Growth after Diagnosed with Cancer
}

\author{
Sook Lee ${ }^{1}$, Yeon Jung $\mathrm{Kim}^{2}$, and Yun Jung $\mathrm{Choi}^{3}$ \\ ${ }^{1}$ Dept. of Nursing, Dankook University, Korea \\ ${ }^{2}$ Dept. of Nursing, Nazarene University, Korea \\ ${ }^{3}$ Red Cross College of Nursing, Chung-Ang University, Korea \\ 2yjkim00@kornu.ac.kr
}

\begin{abstract}
The purpose of this study is to perform the structural equation modeling of relationship among personality, depression, social support, subjective severity, deliberate rumination, intrusive rumination and post-traumatic growth of patients with breast cancer. 201 women participated in this study recruited from inpatients or outpatients female cancer clinics in Korea. Personality, social support, and deliberate rumination had significant positive direct effect on post-traumatic growth. Social support, subjective severity and intrusive rumination had significant indirect effect on posttraumatic growth via deliberate rumination. Depression had significant positive and direct effect on intrusive rumination. The findings of this study suggest a more comprehensive model of growth would be helpful in understanding the various factors which play a role in cancer survivors' perception of psychological growth.
\end{abstract}

Keywords: Posttraumatic, Growth, Depression, Personality, Social, Support

\section{Introduction}

Patients who get a diagnosis of a cancer disease suffer from a variety of problems during their disease process. They undergo a combination of invasive treatments and side effects, strained interpersonal relationships as well as emotional issues such as depression, anxiety and fear of recurrence [1]. However, some patients report positive changes including improved sense of personal strength, satisfaction of relationships with others and realized goals and meanings of their lives in novel perspectives [2][3][4][5][6].

This phenomenon has been identified as "post-traumatic growth" by Tedeschi and Calhoun [7]. Post-traumatic growth (PTG) refers to positive changes in people which goes beyond an ability to resist and not to be damaged by highly stressful circumstances. In research, there is an increasing awareness that a cancer experience is not only viewed as negative but also has a sort of psychological sequel which is beneficial for patients with cancer [8]. For this reason, the identification of factors that are associated with PTG after diagnosed with cancer is of importance.

A variety of correlates of PTG have been identified, including personality traits [9], depression [10][11], social support [12][13][14], intrusive rumination [15], deliberate

Article history:

Received (January 13, 2018), Review Result (February 12, 2018), Accepted (March 20, 2018) 
rumination [3][14][15], stressfulness of cancer [16][17][18] and self-esteem and cancercoping [19]. These factors are comprehensively interrelated with each other concerning the adjustment to life with cancer. It is beneficial to determine causal relationships among factors regarding PTG of patients with cancer.

The purpose of this study was to perform the structural equation modeling (SEM) of relationship: personality, depression, social support, subjective severity, deliberate rumination, intrusive rumination to PTG in female patients with cancer. A more extensive understanding of such people's perspective will be expected.

\section{Method}

\subsection{Theoretical framework}

The Model of Post-traumatic Growth [20] provided the theoretical basis of this study. This model describes that PTG is most likely a result of attempt for psychological adaptation for survival, and it can coexist with the residual distress of the trauma which is considered as ongoing process to adjust oneself from the negative state of emotion. PTG originates from person's character before the traumatic events; optimism, extraversion and openness to experience may be related to posttraumatic growth. These characteristics are thought to play a key role on positive adaptation of patients with cancer from threatening problems [20].

Pérez, et al., [21] reported that patients who concentrated on negative aspect of one's illness spent more time on intrusive rumination, which may lead to higher levels of depressive symptoms. And some researchers have examined the relationship between depression and PTG, reported that depression was associated with lower level of PTG in cancer survivors [10][11].

In particular, Tedeschi and Calhoun [20] have emphasized the important role of support among factors related with PTG. Since social supports from diverse systems provide a new perspective of trauma it could help reform the meaning of the trauma and promote one's growth. Another noteworthy aspect of social support is to minimize a negative rumination which automatically rose along with the emotional distress in the process of PTG.

There is a positive correlation between PTG and social supports from meaningful interpersonal relationships [12][13]. Patients with breast cancer who have a strong intimacy with their spouses show high levels of PTG [19][22]. Coward and Kahn [23] reported women with breast cancer had a motivation from their children to put efforts into an overcome their illnesses. There is an assumption the personality of female patients may change to more active ones and they try to develop more positive perspective of various life-related problems including the battle against the cancer. Female cancer patients show a certain degree of reduction in negative sentiment like loneliness, while increase in interpersonal relationship [23][24][25].

Data from recent studies provide some support for the hypothesized relationships between cognitive process and PTG [26]. Morris and Shakespeare-Finch [14] indicate the deliberate rumination was associated with greater PTG in cancer survivors. Deliberate rumination decreases depression or anxiety of patients with cancer. This is an intentional thought process that active engagement makessense and solves problems. Otherwise, intrusive rumination is linked to increased negative psychological consequences [17][20]. As the traumatic experience is prolonged, deliberate rumination gives positive effect on PTG, however, intrusive rumination in the early stage of traumatic incidents also helps increase in PTG [16]. 
In the initial stage of a trauma, people experience negative emotions driven by intrusive rumination. But they don't stay on this status. They rather undergo the process to consistently contemplate how to understand and overcome the state induced by the trauma [20]. This deliberate rumination is the process for patients with cancer to persistently figure out and chew over to fight against the disease and potential impacts of it, which eventually draws positive meanings out of the illness itinerary [3][14].

Perception of cancer as a traumatic stressor may be more important in predicting PTG than objective conditions such as stages of cancer or side effects from treatments [18]. Some researchers reported that the subjective severity of illness was strongly associated with PTG [17][18]. They suggest the essential role of subjective appraisal in adjustment to cancer. The perception of severity of traumatic events triggers necessities for an active coping mechanism, which plays a role as a precursor to increase the deliberate rumination.

In Summary, the present study focused on the relative contributions of personality, rumination (deliberate and intrusive), depression, social support and subjective severity to PTG by SEM.

\subsection{Sample}

201 women participated in this study. The average age of participants was $51.9(\mathrm{SD}=9.63)$ years, the majority of participants were married $(n=87.1 \%)$. Only $3.5 \%$ had received elementary school education and $43.3 \%$ university. Sites of cancer were breast $(n=123,61.2 \%)$ or ovary and/or uterine $(n=78,48.8 \%)$. The majority of study participants were diagnosed with stage I $(n=66,32.9 \%)$ or stage II $(n=67,33.3 \%)$, and the mean time of illness with cancer was 2.11 years $(\mathrm{SD}=0.55)$.

\subsection{Data collection}

The data were collected from November, 2012 to April, 2013. Participants were recruited from inpatients or outpatients female cancer clinics in Korea. The inclusion criteria were (a) diagnosis of cancer (in ovary, uterine or breast), (b) ages between 18 and 64 years. Before the data collection, ethical approvals were obtained from the institutional review board of Ewha Womans University in Seoul, Korea. To all participants were given a sufficient explanation regarding the study objectives and study processes. Their rights as participants were acknowledged. Only those who voluntarily signed the informed consent were permitted to enter the study.

\subsection{Study tool}

\subsubsection{Post-traumatic growth}

It was originally developed by Tedeschi and Calhoun [7], which was translated and revised into Korean by Song, et al. [27]. It is a self-reporting scale consisted of 16 items with 6-point Likert criteria. Each item was measured on a rating scale 0 to 5, representing 'did not experience this change as a result of my crisis'to 'experience to a very great degree', respectively. Total scores range from 0 to 80 , with higher scores meaning higher levels of posttraumatic growth. The Korean PTGI contains 4 domains subscale: changed perception of self, relating to others, new possibilities, and spiritual change. Cronbach's alpha was .95 in this study. 


\subsubsection{Deliberate and intrusive rumination:}

It was assessed by the Event Related Rumination Inventory (ERRI). The ERRI was developed by Cann, et al. [28], which was translated into Korean by Yoo [29]. The items included either positive or negative forms of repetitive thinking about a highly stressful event. It is a self-reporting scale which consists 20 items with 4-point Likert criteria. Total scores range from 4 to 80 with higher scores meaning higher levels of rumination. ERRI contains 2 domains subscale: deliberate rumination and intrusive rumination. The Cronbach's alpha was .94 for intrusive rumination and .88 for intrusive rumination [28]. In this study, those of Cronbach's alpha were .93 and .92 .

\subsubsection{Personality}

It was assessed by the Myers-Briggs Type Indicator (MBTI). The MBTI was developed by Myers and Briggs [30], which was translated and revised into Korean by Korea MBTI Institute. We used Korean version MBTI form G and extracted part of Extraversion Introversion. In this study, the continuous scoring method described in the MBTI manual [31] was used, which suggests the use of continuous scores in correlational research such as Extraversion-Introversion index. The individual gathers energy from either the outer world (Extraversion "E') or the inner world (Introversion "I'"). This index is scored such that increasingly lower scores indicate increasing Extraversion preference whereas higher scores indicate increasing Introversion preference. Cronbach's alpha was .73 in this study.

\subsubsection{Depression}

It was assessed by the Zung's Depression Inventory (ZDI). The ZDI was developed by Zung [32], which was translated into Korean by Lee [33]. The scale consists of 20 items with 4-point Likert criteria. 20 items are assessing 3 sources of pervasive, physiological, psychological depression. Total scores range from 4 to 80, with higher scores meaning higher levels of depressive mood. Cronbach's alpha for this instrument had been reported to be .84 from previous study [33] and .75 in this study.

\subsubsection{Social support}

It was assessed by The Multidimensional Scale of Perceived Social Support (MSPSS). The MSPSS was developed by Zimet, et al. [34], which was translated into Korean by Shin and Lee [35]. 12 items are assessing 3 sources of support: family, friends and significant others. Items are rated on a 5-point Likert-scale ranging from 1 (very strongly disagree) to 5 (very strongly agree). Total scores range from 5 to 60 , with higher scores indicate a greater support. Cronbach's alpha for this instrument had been reported to be .89 from a previous study [35]. Cronbach's alpha was .86 in this study.

\subsubsection{Participant's perception of cancer diagnosis severity}

It was assessed via an eleven-point Likert scale ranging from 0 (not at all traumatic) to 10 (severely traumatic).

\subsection{Data analysis}

Pearson correlation coefficients were calculated using SPSS 19.0 program (IBM). The SEM was conducted using AMOS 19.0 program to test the proposed model of personality, 
deliberate rumination, intrusive rumination, social support, depression, subjective severity on PTG. Only indicators which were significantly related to PTG were selected for inclusion into the model. we selected variables whose fit indices were high and whose coefficient P-values could be interpreted as significant. Statistical significance was set at $\mathrm{P}<0.05$.

\section{Results}

\subsection{Measurement model}

Factor analysis and reliability of measurement tools for the variables. For personality and subjective severity a single indicator was used, which due to assigning a fixed value the variance of single indicators specified. The data were demonstrated through a Kaiser-MeyerOlkin measure. All indicators were greater than 0.70 (appropriate), suggesting that intrusive rumination, deliberate rumination, depression and social support were useful indicators of PTG. The components accounted for 57.1-78.7\% of variance in the data with a strong loading. These results for the measures with sound psychometric properties above 0.50. Such factor loadings indicated all factors used in this study were supported by proper validity [36].

Table 1. Goodness of fit measures: criteria and acceptable fit interpretation

\begin{tabular}{|c|c|c|c|}
\hline $\begin{array}{c}\text { Goodness of } \\
\text { Fit Measures }\end{array}$ & Results & $\begin{array}{c}\text { Criterion } \\
\text { level }\end{array}$ & Interpretation \\
\hline$\div 2 / \mathrm{df}$ & 1.37 & $1-3$ & \\
\hline GFI & 0.95 & $\geq 0.90$ & \multirow{2}{*}{$\begin{array}{c}\text { The results meet } \\
\text { all the criterion of good } \\
\text { model fit. }\end{array}$} \\
\hline AGFI & 0.91 & $\leq 0.08$ & $\geq 0.90$ \\
\hline SRMR & 0.04 & $\geq 0.90$ & $\leq 0.05$ \\
\hline NFI & 0.95 & 0.98 & \\
\hline RMS & 0.04 & \\
\hline
\end{tabular}

GFI: goodness of fit index, AGFI: adjusted goodness of fit index, SRMR: standardized root means square residual, NFI: normal fit index, CFI: comparative fit index, RMSEA: root mean square residual error of approximation.

\subsection{Model testing}

The initial hypothesized model did not fit empirical data well. So we modified it using the modification indices (MI) and psychological depression has been deleted in this process. Those indicated a good model fit for the entire criterion [Table 1] [37].

Personality $(\hat{\mathrm{a}}=.144, \mathrm{p}<.05)$, social support $(\hat{\mathrm{a}}=.335, \mathrm{p}<.05)$, and deliberate rumination $(\hat{\mathrm{a}}=.408, \mathrm{p}<.01)$ had significant positive direct effect on PTG. And social support $(\hat{\mathrm{a}}=.114$, $\mathrm{p}<.05)$, subjective severity ( $\hat{\mathrm{a}}=.093, \mathrm{p}<.05)$ and intrusive rumination $(\hat{\mathrm{a}}=.177, \mathrm{p}<.01)$ had significant positive indirect effect on PTG. These predictors explained $38.5 \%$ of the variance in PTG of female patients with cancer. And the findings indicated social support ( $\hat{a}=.278$, $\mathrm{p}<.01)$, subjective severity $(\hat{\mathrm{a}}=.210, \mathrm{p}<.01)$ and intrusive rumination $(\hat{\mathrm{a}}=.435, \mathrm{p}<.01)$ had significant direct effect on deliberate rumination and depression( $(\hat{a}=.365, \mathrm{p}<.01)$ had a significant indirect effect on deliberate rumination via intrusive rumination.

These predictors explained $34.1 \%$ of the variance in deliberate rumination of female patients with cancer. Depression $(\hat{\mathrm{a}}=.839, \mathrm{p}<.01)$ had significant direct effect on intrusive rumination and explained $72.5 \%$ of the variance in intrusive rumination of female patients with cancer [Table 2], [Figure 1]. 
Table 2. Standardized direct, indirect and total effects for the model

$S M C=$ Squared multiple correlations $(R 2)$

\begin{tabular}{|c|c|c|c|c|c|}
\hline \multicolumn{2}{|c|}{ Variables } & Direct effect $(p)$ & Indirect effect $(\mathrm{p})$ & Total effect (p) & SMC \\
\hline \multicolumn{2}{|r|}{ Posttraumatic growth } & - & - & - & - \\
\hline • & Personality & $.144 *(.022)$ & - & $.144 *(.022)$ & - \\
\hline - & Depression & $-.295(.227)$ & $.131(.368)$ & $-.163(.137)$ & - \\
\hline - & Social support & $.335 *(.038)$ & $.114 *(.017)$ & $.449 *(.011)$ & - \\
\hline - & Subjective severity & $.069(.471)$ & $.093 *(.026)$ & $.161(.131)$ & .385 \\
\hline - & Deliberate rumination & $.408 * *(.003)$ & & $.408 * *(.003)$ & - \\
\hline • & Intrusive rumination & $-.021(.930)$ & $.177 * *(.001)$ & $.157(.368)$ & - \\
\hline \multicolumn{2}{|r|}{ Deliberate rumination } & - & - & - & - \\
\hline • & Social support & $.278 * *(.006)$ & $.002(.956)$ & $.280 * *(.005)$ & \multirow{4}{*}{.341} \\
\hline • & Subjective severity & $.210 * *(.007)$ & $.020(.571)$ & $.230 * *(.004)$ & \\
\hline - & Intrusive rumination & $.435 * *(.003)$ & & $.435 * *(.003)$ & \\
\hline • & Depression & - & $.365 * *(.004)$ & $.365 * *(.004)$ & \\
\hline \multicolumn{2}{|r|}{ Intrusive rumination } & - & - & - & - \\
\hline • & Depression & $.839 *(.009)$ & - & $.839 *(.009)$ & \multirow{3}{*}{.725} \\
\hline - & Social support & $.004(.964)$ & - & $.004(.964)$ & \\
\hline - & Subjective severity & $.045(.592)$ & - & $.045(.592)$ & \\
\hline
\end{tabular}

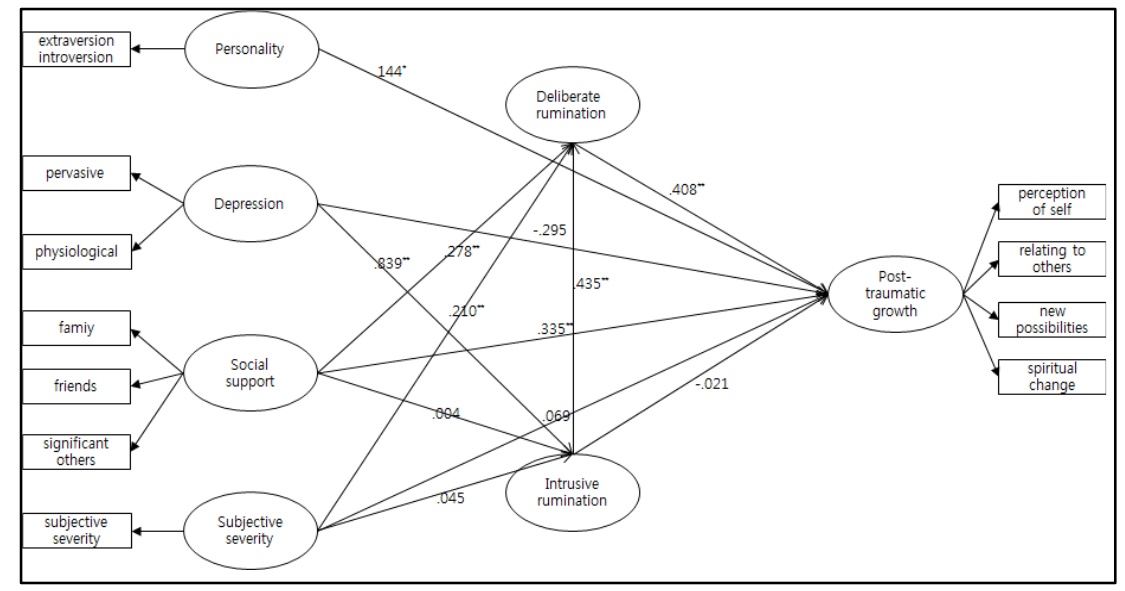

Figure 1. The model of post-traumatic growth

\section{Discussion}

According to this study personality, social support and deliberate rumination have significant positive direct effect on PTG. An extroverted person is sociable [31]. So this person would like to meet people to talk with. Otherwise an introverted person is bashful so they would like to be sunk in meditation alone and prefer to be absorbed in their internal world. Reflecting these characteristics of personality, nursing interventions should be applied 
for two main goals: extroverted cancer patients should be encouraged to share their stories in self-help groups and find a common grounding group activities. For introverted patients, they should be assured to express their emotions and thoughts on cancer experiences while doing activities such as meditation and writing. Personality is not about "good" and "bad", and rather each personality has its own characteristics. Therefore, based on the research outcomes, it is needed to provide nursing intervention customized for each characteristic to promote PTG through self-exposure.

In this study, social support was directly related to PTG of female patients with cancer. This result is consistent with the direct effects of social support supposed previous study [9][14]. According to Tedeschi and Calhoun [7][20], humans who once had gone through traumatic events accept that humans need help from others, try to find the supporting resources around them and want to express their emotions on the trauma. Especially, nurses should include family-oriented supporting systems in their nursing plans, and also help activate self-help groups to share health-related information and disease experiences. In addition, nurses themselves should play the role as a meaningful support system in providing emotional support to cancer patients through utilizing therapeutic communication and helping the patients endure treatment adverse effects and to be confident in the disease fight.

Another important finding is deliberate rumination of female patients with cancer is the major contributing factor. These findings are congruent to previous study [3][14][16]. Antoni, et al. [38] applied the cognitive-behavioral intervention program to female patients with breast cancer in the early stage once in a week for 10 weeks and found stress, depression and intrusive rumination decreased. As well in the meantime optimism and emotional expression increased to support the struggle against cancer disease. Based on those findings, nurses should help the patients with cancer to reflect on them and express their emotions to find the positive meanings in the disease process. Also, cognitive intervention should be given to support a cognitive restructure.

This study shows social support, subjective severity, and intrusive rumination have indirect effects on PTG through deliberate rumination. These findings were supported to study by Tedeschi and Calhoun [20]. They found the indirect effects of social support thorough deliberate rumination contribute to the PTG through restructuring the meaning of the trauma since social support from diverse systems provide a new perspective of the traumatic event. Therefore, like mentioned above, assessment of the supporting systems of cancer patients should be included in the nursing plan.

And subjective severity has indirect effects on PTG via deliberate rumination. This result supports previous researches; Lee, et al. reported female patients with newly diagnosed cancer feel shocked and feared, but their need and affection for lives lead them to actively cope with the cancer [25]; Tedeschi and Calhoun [7][20], Cann, et al. [28] found, as more threat perception on trauma they have, as more cancer patients challenge themselves to deal with the existing diagram. In contrast, Morris and Shakespeare-Finch [14] pointed out a higher subjective severity accelerates intrusive rumination but turns out only to aggravate distress and has no correlation with deliberate rumination. Choi [39] argued too excessively higher threat perception rather interferes intellectual process for growth, and causes psychological confusion. Like these reports, not all preceding investigations show the same research outcomes. Thus, additional research is necessary before making conclusions based on this finding.

Results of this study show an intrusive rumination has no direct effects on posttraumatic growth, but indirect effect via deliberate rumination. Cann, et al. [28] suggests that the two types of rumination coexist throughout the whole disease process: intrusive rumination takes 
up more proportion in the initial stage, however, its portion decreases over the time during the cognitive process. The deliberate rumination gradually increases to cope constructively with human's conscious effort. Previous researchers reported intrusive rumination as a major predictive factor is the prerequisite condition of actively understanding stressful experiences [16][28]. Chan, et al. [3] also reported that female patients with cancer experience deliberate rumination and intrusive rumination in coexistence throughout the whole disease process. Likewise, this result was also in agreement with that obtained in Chan's study. This study found that the two types of rumination have positive relationship one another and intrusive rumination accounted for a substantial proportion of deliberate rumination. And in this research result, it appeared a depression doesn't have influence on the posttraumatic growth. This result is against the research outcome of Ho, Chan, and Ho [11]. They found that lower level of depression is closely connected with the posttraumatic growth. However, it cannot be clearly said that there is no relation between depression and PTG since the deliberate rumination a key contributing factor to the posttraumatic growth, is largely affected by intrusive rumination, and $73 \%$ of intrusive rumination is explained by depression. Moreover, Zung's scale [32], adopted for measuring depression in this study, includes diverse physical responses originated from depression. Since inpatients who are hospitalized for surgery, chemotherapy, and radiation therapy are included in this study, it is thought it is unclear whether the physical response are due to treatment adverse effects or due to depression.

On basis of this research outcome, more reflection on the disease can benefit PTG so that nurses should understand the rumination characteristics of women with cancer. So they are able to plan a cognitive nursing intervention to promote PTG by replacing intrusive rumination which automatically happens in the early stage of diagnosis with deliberate rumination. Furthermore, nursing researchers are expected to figure out the diverse factors affecting the pathway of intrusive and deliberate rumination and conduct the further study on analyzing the factors. Moreover, it is thought that nursing intervention should be provided for various preceding factors to give positive influence on PTG through deliberate rumination.

The findings of this study suggest a more comprehensive model of growth would be helpful in understanding the various factors which play a role in cancer survivors' perception of psychological growth. Nurses and health providers need to assist patients with cancer to comprehend their experience regarding cancer in new perspectives to facilitate the PTG, which requires considering patients' personality, cognitive, emotional, and social factors.

\section{Limitations of the study}

This study was conducted using convenience sampling, which needs careful to be considered regarding the generalization of results as patients with cancer have a variety of factors which are not entirely defined yet.

\section{Acknowledgements}

This work was partially supported by Sigma Theta Tau International Honor Society of Nursing Lambda Alpha Chapter-at-Large.

\section{References}

[1] I. Okamoto, D. Wright, and C. Foster, "Impact of cancer on everyday life: A systematic appraisal of the research evidence,” Health Expectations, vol. 15, no. 1, pp.97-111, (2012) 
[2] S.Y. Hwang, "An exploratory analysis of the existential experiences of women with cancer," Journal of the Korean Neuropsychiatric Association. vol.45, no.6, pp.554-564, (2006).

[3] M.W. Chan, S.M. Ho, R.G. Tedeschi, and C. Leung, "The valence of attentional bias and cancer-related rumination in posttraumatic stress and posttraumatic growth among women with breast cancer," PsychoOncology, vol.20, no.5, pp.544-552, (2011)

[4] F. Mols, A.J. Vingerhoets, J.W.W. Coebergh, and L.V. Van de Poll-Franse, "Well-being, posttraumatic growth and benefit finding in long-term breast cancer survivors," Psychology Health, vol.24, no.5, pp.583595, (2009)

[5] S M. Silva, C. Crespo, and M.C. Canavarro, "Pathways for psychological adjustment in breast cancer: A longitudinal study on coping strategies and posttraumatic growth," Psychology Health, vol.27, no.11, pp.1323-1341, (2012)

[6] D.M. Posluszny, A. Baum, R.P. Edwards, and M.A. Dew, "Posttraumatic growth in women one year after diagnosis for gynecologic cancer or benign conditions," Psychosocial Oncology, vol.29, no.5, pp.561-572, (2011)

[7] R.G. Tedeschi and L.G. Calhoun, "The posttraumatic growth inventory: Measuring the positive legacy of trauma," Journal of Traumatic Stress, vol.9, pp.455-471, (1996)

[8] I.J. Lee, "A systematic review of posttraumatic growth in cancer," Social Work Practice \& Research, vol.6, pp.81-113, (2009)

[9] H.J. Kim, J.H. Kwon, J.N. Kim, R. Lee, and K.S. Lee, "Posttraumatic growth and related factors in breast cancer survivors," The Korean Journal of Health Psychology, vol.13, no.3, pp.781-799, (2008)

[10] K. Mystakidou, E. Tsilika, E. Parpa, D. Kyriakopoulos, N. Malamos, and D. Damigos, "Personal growth and psychological distress in advanced breast cancer," Breast, vol.17, no.4, pp.382-386, (2008)

[11] S.M.Y. Ho, C.L.W. Chan, and R.T.H. Ho, "Posttraumatic growth in Chinese cancer survivors," PsychoOncology, vol.13, pp.377-389, (2004)

[12] I.Y. Han and I.J. Lee, "Posttraumatic growth in patients with cancer," Korean Journal of Social Welfare Studies, vol.42, no.2, pp.419-441, (2011)

[13] O. Bozo, E. Gündogdu, and C. Büyükasik-Colak, "The moderating role of different sources of perceived social support on the dispositional optimism-posttraumatic growth relationship in postoperative breast cancer patients," Journal of Health Psychology, vol.14, no.7, pp.1009-1020, (2009)

[14] B.A. Morris, and J. Shakespeare-Finch, "Rumination, post-traumatic growth, and distress: Structural equation modelling with cancer survivors," Psycho-Oncology, vol.20, pp.1176-1183, (2011)

[15] L.G. Calhoun, A. Cann, R.G. Tedeschi, and J. McMillan, "A correlational test of the relationship between posttraumatic growth, religion, and cognitive procession," Journal of Traumatic Stress, vol.13, no.3, pp.521257, (2000)

[16] K. Taku, A. Cann, R.G. Tedeschi, and L.G. Calhoun, "Intrusive versus deliberate rumination in posttraumatic growth across US and Japanese samples," Anxiety, Stress \& Coping, vol.22, no.2, pp.129-136, (2009)

[17] K.M. Bellizzi and T.O. Blank, "Predicting posttraumatic growth in breast cancer survivors," Health Psychology, vol.25, no.1, pp.47-56, (2006)

[18] M.J. Cordova, J. Giese-Davis, M. Golant, C. Kronenwetter, and V. Chang, "Breast Cancer as trauma: Posttraumatic stress and posttraumatic growth,” Journal of Clinical Psychology in Medical Settings. vol.14, no.4, pp.309-319, (2007)

[19] S. Lee and Y.J. Kim, "Posttraumatic growth of patients with breast cancer," Journal of Korean Academy of Nursing, vol.42, no.6, pp.907-915, (2012)

[20] R.G. Tedeschi and L.G. Calhoun, "Posttraumatic growth: Conceptual foundation and empirical evidence," Psychological Inquiry, vol.15, no.1, pp.1-18, (2004) 
[21] J.E. Pérez, A.R. Smith, R.L. Norris, K.M. Canenguez, E.F. Tracey, and S.B. DeCristofaro, "Types of prayer and depressive symptoms among cancer patients: The mediating role of rumination and social support," Journal of Behavioral Medicine, vol.34, no.6, pp.519-530, (2011)

[22] T. Weiss, "Correlates of posttraumatic growth in married breast cancer survivors," Journal of Social Clinical Psychology, vol.23, pp.733-746, (2004)

[23] D. Coward and D. Kahn D, "Transcending breast cancer: Making meaning from diagnosis and treatment," Journal of Holistic Nursing, vol.23, pp.264-283, (2005)

[24] Y.H. Noh, "Breast cancer survivor's conquest experience," Journal of Qualitative Research, vol.4, no.1, pp.7$25,(\mathbf{2 0 0 3})$

[25] S.H. Lee, J.E. Kim, H.K. Lee, N.M. Kang, H.W. Kim, E.H. Lee, M.H. Hur, and Y.S. Park, "A groundedtheory approach to the process of life adaptation in women with cervical cancer," Journal of Korean Academy of Women's Health Nursing, vol.10, no.1, pp.32-41, (2004)

[26] L.G. Calhoun and R.G. Tedeschi, "Posttraumatic growth: Future directions Posttraumatic growth: Positive change in the aftermath of crisis," NJ: Lawrence Erlbaum Associates, Inc., pp.215-238, (1998)

[27] S.H. Song, H.S. Lee, J.H. Park, and K.H. Kim, "Validity and reliability of the Korean version of the posttraumatic growth inventory," Korean Journal of Health Psychology, vol.14, no.1, pp.193-214, (2009)

[28] A. Cann, L.G. Calhoun, R.G. Tedeschi, K.N. Triplett, T. Vishnevsky, and C.M. Lindstrom, "Assessing posttraumatic cognitive processes: The event related rumination inventory,” Anxiety, Stress \& Coping, vol.24, no.2, pp.137-156, (2011)

[29] H.J. Yoo, "A study of the structural relationship between variables that influence posttraumatic growth," $\mathrm{Ph}$. D. dissertation, Pusan University, Pusan, Korea, (2012)

[30] L.B. Myers, "Manual: The Myers-Briggs type indicator," Princeton, NJ: Educational Testing Services, (1962)

[31] J.T. Kim, H.S. Sim, and S.B. Je, Theory, psychometrics, application MBTI, (2nd edition), Assesta, Seoul, (2009)

[32] W.W.K. Zung, “A self-rating depression scale,” Arch General Psychiatry, vol.12, pp.63-70, (1965)

[33] J.H. Lee, "Development of the Korean form of Zung's self-rating depression scale," The Yeungnam University Medical Journal, vol.12, no.2, pp.292-305, (1995)

[34] G.D. Zimet, N.W. Dahlem, S.G. Zimet, and G.K. Farley, "The multidimensional scale of perceived social support," Journal of Personality Assessment, vol.52, pp.30-41, (1988)

[35] C.S. Shin and Y.B. Lee, "The effects of social supports on psychosocial well-being of the unemployed," Korean Journal of Social Welfare, vol.37, pp.241-269, (1999)

[36] H.S. Lee and J.H. Lim, Structural equation modeling \& AMOS 18.0/19.0, Jyphyunjae, Seoul, (2011)

[37] B.R. Bae, "Structural Equation Modeling with Amos 19," Principles and Practice, Chungram, Seoul, (2011)

[38] M.H. Antoni, J.M. Lehman, K.M. Kilbourn, A.E. Boyers, J.L. Culver, S.M. Alferi, S.E. Yount, B.A. McGregor, P.L. Arena, S.D. Harris, and C.S. Carver, "Cognitive-behavioral stress management intervention decreases the prevalence of depression and enhances benefit finding among women under treatment for earlystage breast cancer," Health Psychology, vol.20, no.1, pp.20-32, (2001)

[39] S.M. Choi, "Exploration of posttraumatic growth related variables," Ph. D. dissertation, Korea University, Seoul, Korea, (2008) 